PROCEEDINGS OF THE

AMERICAN MATHEMATICAL SOCIETY

Volume 133, Number 4, Pages 1231-1237

S 0002-9939(04)07662-2

Article electronically published on September 16, 2004

\title{
THE RATIO OF THE LENGTH OF THE UNIT CIRCLE TO THE AREA OF THE UNIT DISC IN MINKOWSKI PLANES
}

\author{
ZOKHRAB MUSTAFAEV
}

(Communicated by Jon G. Wolfson)

\begin{abstract}
In their paper "An Introduction to Finsler Geometry," J. C. Alvarez and C. Duran asked if there are other Minkowski planes besides the Euclidean for which the ratio of the Minkowski length of the unit "circle" to the Holmes-Thompson area of the unit disc equals 2. In this paper we show that this ratio is greater than 2 , and that the ratio 2 is achieved only for Minkowski planes that are affine equivalent to the Euclidean plane. In other words, the ratio is 2 only when the unit "circle" is an ellipse.
\end{abstract}

\section{INTRODUCTION}

In this paper we give a surprisingly simple proof that if $B$ is the unit disc in a two-dimensional Minkowski space $(X,\|\cdot\|)$ and $\mu$ represents Minkowski measure of the appropriate dimension as defined below, then

$$
2 \leq \frac{\mu_{B}(\partial B)}{\mu_{B}(B)} \leq \pi
$$

with equality on the left if and only if $B$ is an ellipse and equality on the right if and only if $B$ is a parallelogram.

In their paper [3], R. D. Holmes and A. C. Thompson investigated the ratio of

$$
\omega(B)=\frac{\varepsilon_{d-1}}{d \varepsilon_{d}} \frac{\mu(\partial B)}{\mu(B)},
$$

where $\varepsilon_{d}=\pi^{d / 2} \Gamma(d / 2+1)^{-1}$ and is the volume of a $d$-dimensional Euclidean unit ball. They established certain bounds on $\omega$ which state that if $B$ is a $d$-dimensional Minkowski unit ball, then

$$
\frac{1}{2} \leq \omega(B) \leq \frac{d}{2}
$$

with equality on the right if $B$ is a cube or an "octahedron". When $d=2$, we obtain

$$
\frac{1}{2} \leq \omega(B)=\frac{1}{\pi} \frac{\mu(\partial B)}{\mu(B)} \leq 1
$$

Holmes and Thompson posed the question, "What is the lower bound for $\omega(B)$ in $R^{d}$ ?" Our main result provides the answer to this question for the case $d=2$.

Received by the editors October 28, 2003 and, in revised form, December 15, 2003.

2000 Mathematics Subject Classification. Primary 52A10, 52A40.

(C) 2004 American Mathematical Society Reverts to public domain 28 years from publication 
We shall also prove a stronger result involving the inner and outer radii of the unit circle.

We recommend the interesting book by Thompson "Minkowski Geometry" for a thorough discussion on this topic.

\section{Definitions}

A Minkowski space is a pair $(X,\|\cdot\|)$, where $X$ is finite dimensional and $\|\cdot\|$ is a norm. It is sometimes desirable to consider $X$ as $R^{d}$ equipped with an arbitrary norm. The unit ball $B=B[0,1]$ in $(X,\|\cdot\|)$ is the set

$$
B:=\{x \in X:\|x\| \leq 1\} .
$$

The unit sphere in $(X,\|\|$.$) is the boundary of the unit ball, which is denoted by$ $\partial B$. In a two-dimensional space, we will call the unit ball and the unit sphere, respectively, the unit disc and the unit circle.

The function $h_{K}$ defined by

$$
h_{K}(f):=\sup \{f(x): x \in K\}
$$

is called the support function of $K$.

We shall suppose that $X$ also possesses the standard Euclidean structure and that $\lambda$ is the Lebesgue measure induced by that structure. We refer to this measure as volume (area) and denote it as $\lambda($.$) . The volume \lambda$ gives rise to a dual volume $\lambda^{*}$ on the convex subsets of $X^{*}$.

We identify $R^{d}$ and $R^{d *}$ by using the standard basis. In that case, $\lambda$ and $\lambda^{*}$ coincide in $R^{d}$.

Definition. Let $X$ be a two-dimensional Minkowski space and $B$ be the unit disc in $X$. The Holmes-Thompson area for the convex body $K$ in $X$ is defined by the equation

$$
\mu_{B}(K)=\frac{\lambda(K) \lambda^{*}\left(B^{\circ}\right)}{\pi} .
$$

This definition coincides with the standard notion of area if the plane is Euclidean.

Recall that if $\gamma:[a, b] \rightarrow R^{2}$ is a regular parametrized curve in the Minkowski plane $\left(R^{2},\|\cdot\|\right)$, then the Minkowski length of $\gamma$ is defined to be $\int_{a}^{b}\left\|\gamma^{\prime}(t)\right\| d t$.

We shall denote the Minkowski length of a curve $c$, measured with respect to a unit ball $B$, as $\mu_{B}(c)$.

Let $\left(R^{2},\|\cdot\|\right)$ be a two-dimensional Minkowski space whose unit ball is $B$. We define the convex body $I_{B}$ as the polar reciprocal of $B$, with respect to the Euclidean unit circle, rotated through $90^{\circ}$. It turns out that $I_{B}$ plays a central role for the solution of the isoperimetric problem in the Minkowski plane. More precisely, the convex bodies with the minimum Minkowski perimeter for a given enclosed area are the translates of $I_{B}$. The same applies, dually, for bodies of maximal area for a given perimeter. For more details see ([6], pp. 119-121]). Among the homothetic images of $I_{B}$, we want to specify a unique one. We call this the isoperimetrix, denoted by $\hat{I}_{B}$.

Definition. The isoperimetrix for the Holmes-Thompson area is defined by

$$
\hat{I}_{B}=\frac{\pi}{\lambda^{*}\left(B^{\circ}\right)} I_{B}
$$


This normalization insures that $\mu_{B}\left(\partial \hat{I}_{B}\right)=2 \mu_{B}\left(\hat{I}_{B}\right)$ (see proof below). From this point on, $\hat{I}_{B}$ will stand for this definition.

Note that $\mu_{B}\left(\hat{I}_{B}\right)=\pi$, since $\lambda^{*}\left(B^{\circ}\right)=\lambda\left(I_{B}\right)$.

When $d=2$ we can define the mixed volume of two convex bodies $K_{1}$ and $K_{2}$ as

$$
V\left(K_{1}, K_{2}\right)=2^{-1} \int_{\partial K_{1}} h_{K_{2}}\left(f_{x}\right) d s(x)=2^{-1} \int_{\partial K_{2}} h_{K_{1}}\left(f_{x}\right) d s(x)
$$

(see [6], p. 59, when $d=2$ ), where $f_{x}$ is a Euclidean unit vector in $X^{*}$. Using this formula we can write the Minkowski length of the boundary of $K$ in terms of the mixed volume:

Proposition. If $K$ is a convex body in $X$, then

$$
\mu_{B}(\partial K)=\int_{\partial K} h_{I_{B}}\left(f_{x}\right) d s(x)=2 V\left(K, I_{B}\right) .
$$

If $\partial K$ does not have a smooth boundary, then the set of points for which $\partial K$ is not differentiable is at most countable, and this set will not affect the values of the integrals. Therefore, these formulas are true for all convex bodies $K$.

Proposition. If $\hat{I}_{B}$ is the isoperimetrix defined as above in a Minkowski plane $(X, B)$, then

$$
\mu_{B}\left(\partial \hat{I}_{B}\right)=2 \mu_{B}\left(\hat{I}_{B}\right)
$$

i.e., the Minkowski length of $\hat{I}_{B}$ is 2 times the Minkowski area of $\hat{I}_{B}$.

Proof. Using the definition of $\hat{I}_{B}$ and $\mu_{B}\left(\partial I_{B}\right)=2 \lambda\left(I_{B}\right)$, we obtain

$$
\begin{gathered}
\mu_{B}\left(\partial \hat{I}_{B}\right)=\frac{\pi}{\lambda^{*}\left(B^{\circ}\right)} \mu_{B}\left(I_{B}\right)=2 \frac{\pi}{\lambda^{*}\left(B^{\circ}\right)} \lambda\left(I_{B}\right) \\
=2 \frac{\lambda^{*}\left(B^{\circ}\right) \lambda\left(\hat{I}_{B}\right)}{\pi}=2 \mu_{B}\left(\hat{I}_{B}\right) .
\end{gathered}
$$

Definition. If $K$ is a convex body in $X$, the inner radius of $K, r(K)$ is defined by

$$
r(K):=\max \left\{\alpha: \exists x \in X \text { with } \alpha \hat{I}_{B} \subseteq K+x\right\},
$$

and the outer radius of $K, R(K)$ is defined by

$$
R(K):=\min \left\{\alpha: \exists x \in X \text { with } \alpha \hat{I}_{B} \supseteq K+x\right\} .
$$

\section{NEEDED THEOREMS}

Theorem 1 (Blaschke-Santaló (see [6], p. 54)). If $K$ is a d-dimensional symmetric convex body, then

$$
\lambda(K) \lambda^{*}\left(K^{\circ}\right) \leq \varepsilon_{d}^{2}
$$

where $\varepsilon_{d}$ is the volume of a d-dimensional Euclidean unit ball. Moreover, equality occurs if and only if $K$ is an ellipsoid.

Theorem 2 (Mahler-Reisner (see [4] or 6], p. 54)). If $K$ is a d-dimensional zonoid with nonempty interior, then

$$
\frac{4^{d}}{d !} \leq \lambda(K) \lambda^{*}\left(K^{\circ}\right)
$$

Moreover, equality occurs if and only if $K$ is a parallelotope. In the case when $d=2$, all symmetric convex bodies are zonoids. 
Theorem 3 (Minkowski inequality for mixed volumes (see [5], p. 317 or [6], p. 57)). If $K_{1}$ and $K_{2}$ are $d$-dimensional convex bodies, then

$$
V^{d}\left(K_{1}[d-1], K_{2}\right) \geq \lambda\left(K_{1}\right)^{d-1} \lambda\left(K_{2}\right) .
$$

Equality holds if and only if $K_{1}$ and $K_{2}$ are homothetic.

Theorem 4 (Gołąb (see 6], pp. 113-114)). If B is the unit ball in a two-dimensional Minkowski space, then $6 \leq \mu_{B}(\partial B) \leq 8$ with equality on the left if and only if $B$ is an affine regular hexagon and equality on the right if and only if $B$ is a parallelogram.

Theorem 5 (see [2], p. 108). If $K_{1}$ and $K_{2}$ are Minkowski plane convex bodies, and $K_{2}$ is inscribed in $K_{1}$, then the mixed areas of $K_{1}$ and $K_{2}$ is greater than or equal to the arithmetic mean of the areas of $K_{1}$ and $K_{2}$.

\section{MAin Results}

By the Blaschke-Santaló and the Mahler-Reisner inequalities (Theorems 1 and 2) we have

$$
8 \leq \lambda(B) \lambda^{*}\left(B^{\circ}\right) \leq \pi^{2} .
$$

That is,

Since

$$
\frac{8}{\pi} \leq \mu_{B}(B) \leq \pi .
$$

we obtain

$$
6 \leq \mu_{B}(\partial B) \leq 8,
$$

$$
\frac{6}{\pi} \leq \frac{\mu_{B}(\partial B)}{\mu_{B}(B)} \leq \pi
$$

The upper extreme value is achieved if $B$ is a parallelogram, which follows from the Blaschke-Santaló and Gołąb Theorems. Obviously, this ratio cannot achieve the lower extreme value, since if $\frac{\mu_{B}(\partial B)}{\mu_{B}(B)}=\frac{6}{\pi}$, then we would have $\mu_{B}(\partial B)=6$, which means $B$ is a regular hexagon and $\mu_{B}(B)=\pi$, which means $B$ is an ellipse.

Therefore,

$$
\frac{6}{\pi}<\frac{\mu_{B}(\partial B)}{\mu_{B}(B)} \leq \pi
$$

Now if we regard $I_{B}$ as $B^{\circ}$ rotated, then $\lambda\left(I_{B}\right)=\lambda^{*}\left(B^{\circ}\right)$. From the BlaschkeSantaló Inequality (Theorem 1) we know that $\lambda(B) \lambda^{*}\left(B^{\circ}\right) \leq \pi^{2}$. Then $\frac{\lambda(B) \lambda^{*}\left(B^{\circ}\right)}{\pi^{2}} \leq$ 1 , and multiplying both sides again by $\lambda(B) \lambda^{*}\left(B^{\circ}\right)$, we obtain

$$
\begin{aligned}
& \mu_{B}^{2}(B)=\frac{\lambda^{2}(B) \lambda^{*^{2}}\left(B^{\circ}\right)}{\pi^{2}} \leq \lambda(B) \lambda^{*}\left(B^{\circ}\right) \\
& =\lambda(B) \lambda\left(I_{B}\right) \leq V^{2}\left(B, I_{B}\right)=\left(\frac{1}{2} \mu_{B}(\partial B)\right)^{2},
\end{aligned}
$$

where the last inequality is derived from the Minkowski inequality for mixed volumes (Theorem 3 ) when $d=2$. The first equality holds if and only if $B$ is an ellipse because of the Blaschke-Santaló Theorem. Then the second equality will hold, since $B$ and $I_{B}$ are homothetic. Thus, we have

$$
\mu_{B}(B) \leq \frac{1}{2} \mu_{B}(\partial B) .
$$

We summarize this discussion in the following theorem. 
Theorem 6. If $B$ is the unit disc in a two-dimensional Minkowski space $(X,\|\cdot\|)$, then

$$
2 \leq \frac{\mu_{B}(\partial B)}{\mu_{B}(B)} \leq \pi
$$

with equality on the left if and only if $B$ is an ellipse and equality on the right if and only if $B$ is a parallelogram.

We can obtain a more precise result using the inner or outer radius of the unit disc in a two-dimensional Minkowski space.

Lemma. If $r(B)$ is the inner radius of the unit disc of $B$, then

$$
r(B) \leq 1
$$

with equality if and only if $B$ is an ellipse.

Proof. Since $r(B) \hat{I}_{B}$ is inscribed in $B$, we have $\lambda\left(r(B) \hat{I}_{B}\right) \leq \lambda(B)$. That is,

$$
r(B)^{2} \frac{\pi^{2}}{\lambda^{2}\left(B^{\circ}\right)} \lambda\left(I_{B}\right) \leq \lambda(B) .
$$

Thus, we obtain

$$
r^{2}(B) \pi^{2} \leq \lambda(B) \lambda\left(B^{\circ}\right) .
$$

By the Blashke-Santaló Theorem, this inequality can only be true if $r(B) \leq 1$. When $r(B)=1$ this inequality is valid only if $B$ is an ellipse.

Lemma. If $R(B)$ is the outer radius of the unit disc of $B$, then

$$
R(B) \geq \frac{3}{\pi}
$$

with equality if and only if $B$ is a regular hexagon.

Proof. Since $B \subseteq R I_{B}$ we have

$$
\mu\left(\partial R \hat{I}_{B}\right) \geq \mu(\partial B) \geq 6 .
$$

Thus,

$$
R \mu_{B}\left(\partial \hat{I}_{B}\right) \geq 6
$$

The result follows from the fact that $\mu_{B}\left(\partial \hat{I}_{B}\right)=2 \pi$.

Obviously if equality holds, then $B$ is a regular hexagon. If $B$ is a regular hexagon, then $B$ and $\hat{I}_{B}$ are homothetic. Thus, $B=R \hat{I}_{B}$ and we obtain $R=\frac{3}{\pi}$.

Remark. From $R(B)=1$, it does not follow that $B$ is an ellipse.

Theorem 7. If $r(B)$ and $R(B)$ are the inner and outer radii of the unit disc of $B$, respectively, then

$$
\begin{aligned}
\text { i) } \frac{\mu_{B}(\partial B)}{\mu_{B}(B)} \geq r+\frac{1}{r}, \\
\text { ii) } \frac{\mu_{B}(\partial B)}{\mu_{B}(B)} \geq R+\frac{1}{R}
\end{aligned}
$$

with equality if and only if $B$ is an ellipse.

Proof. We will prove the first inequality. Proof of the second inequality is the same. 
Taking $K_{2}=r \hat{I}_{B}$ and $K_{1}=B$ in Theorem 8 , we have

$$
2 V\left(B, r \hat{I}_{B}\right) \geq \lambda(B)+\lambda\left(r \hat{I}_{B}\right) .
$$

That is,

$$
2 r \frac{\pi}{\lambda\left(B^{\circ}\right)} V\left(B, I_{B}\right) \geq \lambda(B)+r^{2} \frac{\pi^{2}}{\lambda^{2}\left(B^{\circ}\right)} \lambda\left(I_{B}\right) .
$$

Multiplying both sides by $\frac{\lambda\left(B^{\circ}\right)}{\pi}$, and using the fact that $\lambda\left(B^{\circ}\right)=\lambda\left(I_{B}\right)$, we obtain

$$
r \mu_{B}(\partial B) \geq \mu_{B}(B)+r^{2} \pi .
$$

Thus, we have

$$
\frac{\mu_{B}(\partial B)}{\mu_{B}(B)} \geq \frac{1}{r}+r \frac{\pi}{\mu_{B}(B)}
$$

Recalling that $\frac{\pi}{\mu_{B}(B)} \geq 1$ by the Blashke-Santaló Theorem, we obtain the first inequality. The Blashke-Santaló Theorem also tells us that the equality sign holds if and only if $B$ is an ellipse.

Note that if $R r \leq 1$, then $r+\frac{1}{r} \geq R+\frac{1}{R}$, and if $R r \geq 1$, then $r+\frac{1}{r} \leq R+\frac{1}{R}$.

Corollary. If $r(B)$ and $R(B)$ are inner and outer radii of the unit disk $B$, respectively, then

$$
\begin{gathered}
\text { i) } \frac{\pi-\sqrt{\pi^{2}-4}}{2} \leq r(B) \leq 1, \\
\text { ii) } \frac{3}{\pi} \leq R(B) \leq \frac{\pi+\sqrt{\pi^{2}-4}}{2} \\
\text { iii) } R(B)-r(B) \leq \frac{4 \sqrt{2}}{\pi} .
\end{gathered}
$$

Proof. Since $\frac{\mu_{B}(\partial B)}{\mu_{B}(B)} \leq \pi$ the first and second inequalities can be deduced easily from Theorem 7 and the previous lemmas.

The last inequality can be obtained from Bonnesen's Inequality, which states

$$
\pi^{2}(R(B)-r(B))^{2} \leq \mu_{B}(\partial B)^{2}-4 \pi \mu_{B}(B) .
$$

Now we need to use the Gołab and Mahler-Reisner Theorems on the right side of this inequality to obtain inequality iii).

The right side of the first and the left side of the second inequalities are sharp. It is also desirable to find the sharp boundaries for the remaining inequalities.

\section{ACKNOWLEDGMENT}

I would like to thank Professor Michael Gage, my advisor, for his help and valuable suggestions.

\section{REFERENCES}

[1] Alvarez, J. and Duran, C., "An introduction to Finsler Geometry", Notas de la Escuela Venezolana de Mathematicas, 1998.

[2] Eggleston, H. G., "Convexity", Cambridge Univ. Tracts in Math. and Math. Phys. 47, Cambridge (1958). MR0124813 (23:A2123)

[3] Holmes, R. D. and Thompson, A. C., n-dimensional area and content in Minkowski spaces, Pacific J. Math. 85 (1979), 77-110. MR0571628(81k:52023)

[4] Reisner, S., Zonoids with minimal volume product, Math. Z. 192 (1986), 339-346. MR0845207 (87g:52022) 
[5] Schneider, R., "Convex Bodies: The Brunn-Minkowski Theory", Encyclopedia of Math. and Its Appl. 44, Cambridge Univ. Press, Cambridge (1993). MR1216521 (94d:52007)

[6] Thompson, A. C., "Minkowski Geometry", Encyclopedia of Math. and Its Appl., Vol.63, Cambridge Univ. Press, Cambridge, 1996. MF1406315 (97f:52001)

Department of Mathematics and Computer Science, 1212 Williams Hall, Ithaca ColLEGe, IthacA, New YoRK 14850

E-mail address: zmustafaev@ithaca.edu 